\title{
EDUCARE ALLA SCELTA DELLA SCUOLA SECONDARIA DI SECONDO GRADO E ORIENTARE PER PREPARARE AL FUTURO
}

\author{
Margherita Fontana \\ Dottoranda di Ricerca Università di Extremadura, Badajoz \\ marghfont@yahoo.it
}

Fecha de Recepción: 2 Marzo 2018

Fecha de Admisión: 10 Abril 2018

\section{RIASSUNTO}

L'orientamento è un fattore strutturale di ogni processo formativo, realizzato lungo l'arco di tutta la vita; nella scuola si trovano gli spazi e le opportunità per formarsi e prepararsi alla scelta di una professione futura. Naturalmente la scuola deve acquisire la consapevolezza di ciò, tuttavia in questi anni, in cui le riforme scolastiche si sono succedute, è cresciuta la necessità di far acquisire all'orientamento il compito educativo che mette al centro l'interesse degli studenti e delle studentesse e la maturazione della loro personalità (Del Core, 2008).

L'orientamento scolastico deve indirizzare gli alunni ad esplorare ed acquisire elementi fondamentali per iniziare la costruzione di una propria identità, partendo dalle competenze acquisite.

Fondamentale diventa l'aiuto a riconoscere le competenze possedute, non solo per sostenere la motivazione ma anche per individuare e definire le scelte formative e di lavoro.

È utile nel processo di orientamento, in particolare nella verifica della scelta fatta, riflettere sulle interconnessioni tra caratteristiche personali, specificità del percorso scolastico e caratteristiche delle figure professionali collegate.

"Uno dei principali obiettivi della scuola è fornire agli studenti gli strumenti intellettivi, le convinzioni e le abilità auto-regolatorie che servono loro per autoistruirsi tutta la vita". Imparare ad autogestirsi in situazioni diverse e anche dopo la scuola è il punto di forza che aiuterà gli adolescenti ad essere autonomi e ad "arrivare fino in fondo" (Bandura, 2012, p. 24).

Parole chiavi: orientamento; scelta scuola superiore; autostima; successo scolastico; progetto di vita

\section{ABSTRACT \\ Educate the choice of secondary school of second grade and orientate to prepare for the futu- re. \\ Orientation is a structural factor of all learning process, it is fulfilled lifelong. At school, spaces}


and opportunities to train and to get ready for choosing a future profession are found. School, of course, has to acquire the consciousness of that; nevertheless during the last few years, in which education reforms have followed, it is grow the need to perfuse an educative role at the orientation, so students and their personalities should be the main focus of interest (Del Core, 2008).

School Orientation has to direct students to investigate and to acquire essential elements to start their own identity construction, starting from skills they have acquired before.

So, it becomes essential give a helping hand to all students to recognize the skills owned, not only to support the motivation but also to identify and define the educational and work choices.

In the learning process, particularly during the analysis of the choices done, is useful reflect about the interconnection among personal features, detail of the school process and features of the professional figure related.

"One of the school main purpose is to provide all students with the intellective instruments, conceptions and self-regulatory abilities, useful to their own lifelong learning process". Learning SelfManagement in different situations and, also, after school, is the strong point, which will help students to be independent and "to rich the bottom". (Bandura, 2012, p. 24).

Keywords: guidance; high school choice; self-confidence; scholastic success; life project

\section{INTRODUZIONE}

L'azione orientativa della scuola, dunque, in sinergia con le altre istituzioni formative, a partire dalla famiglia, ha come fine quello di rendere i giovani protagonisti dei momenti decisionali, nell'attuazione del loro individuale progetto di vita, che deve essere autonomamente elaborato e assunto gradualmente, con convinzione e responsabilità.

Le linee guida nazionali per l'orientamento permanente promuovono un sistema di "orientamento centrato sulla persona e sui suoi bisogni, finalizzato a prevenire e contrastare il disagio giovanile e favorire la piena occupabilità, l'inclusione sociale e il dialogo interculturale" (MIUR, 2014): si vuole quindi agevolare l'intraprendenza del soggetto per il suo sviluppo fisico, psichico e intellettuale, affinché sia nelle condizioni di definire e conquistare la propria identità di fronte agli altri e di rivendicare un proprio ruolo nella realtà sociale, culturale e professionale.

C'è unanimità di giudizio sul fatto che è stato restrittivo disporre le attività di orientamento soltanto nella fase di passaggio dalla scuola secondaria di primo grado alle scelte relative alla scuola superiore. Anche se ci si trova di fronte a individui, come i preadolescenti che vivono di fatto, il dramma di dover scegliere il percorso scolastico successivo, nonostante non possiedono una maturità e una capacità decisionale sufficiente per fare una scelta adeguata. La capacità del preadolescente di operare scelte realistiche nell'immediato e nel futuro, per sviluppare e maturare un progetto di vita personale, dipende dal consolidamento di capacità decisionali basate su un'autentica conoscenza di sé, oltre che su un valido percorso educativo, che valorizzi e continui ad elevare le capacità, gli interessi e le attitudini di ogni studente (Del Core, 2008). L'orientamento, dunque, viene sempre più considerato come un processo educativo continuo, cui le varie strutture non formali e informali del territorio, nonché i diversi gradi di scuola successivi, debbono concorrere unitariamente.

Oggi giorno fare delle scelte sia in ambito scolastico che lavorativo è più problematico rispetto al passato. Sembra un paradosso, ma quando ci si trova a dover scegliere dinanzi a più alternative ed opzioni, diventa complicato orientarsi.

Se da un lato, le opportunità di scelta, rispetto al passato, sono notevolmente aumentate, non sono migliorati in corrispondenza, i riferimenti etici ed educativi che sostengono i processi decisionali, con la conseguenza che il momento della scelta diventa difficile da affrontare (Ibidem). 
Viviamo in un'epoca molto complicata, nella quale è davvero difficile prevedere, suggerire o consigliare opzioni, se non quella di considerare l'incertezza come possibile opportunità. La scelta della scuola superiore per lo studente, in questo scenario, delinea una prova evolutiva in cui per la prima volta sperimenta e scopre un'autonomia che gli permette di mettere in atto le nuove capacità riflessive e valutative. Queste capacità, però, sono in fase di maturazione e quindi è molto complicato compiere delle scelte proiettate nel futuro (Mancinelli e Manto, 2004).

"Gli adolescenti devono gestire contemporaneamente dei grossi cambiamenti di tipo biologico, scolastico e sociale" (Bandura, 2012, p. 17).

La scelta della scuola secondaria è un momento importante nella vita degli adolescenti, è una decisione spesso sofferta, che condiziona il futuro scolastico e lavorativo in un periodo difficile e delicato com'è l'adolescenza. Questa scelta, come anche quella di cosa fare dopo la scuola superiore, è un processo complesso che implica fattori basilari, come gli interessi e le inclinazioni specifiche degli studenti, ma che viene influenzato anche da altri fattori, non evidenti, come l'ambiente sociale in cui gli adolescenti vivono. Gli studenti molto spesso, infatti, vengono spinti dai genitori a scegliere un dato percorso di studi, con l'intento di indirizzarli, magari, a seguire lo stesso percorso professionale e lavorativo del padre o della madre. Un ruolo non indifferente nel processo di scelta svolge anche il gruppo dei pari: è inevitabile, infatti, che gli adolescenti si confrontino con le scelte fatte degli amici e di conseguenza si facciano influenzare.

L'orientamento non può scindersi dall'educazione e dall'insegnamento, la scuola, pertanto, deve prevedere, al suo interno, un sistema di supporto all'orientamento, una task force, con l'obiettivo di sviluppare delle politiche in materia di orientamento per tutti i livelli e le aree coinvolte. Del gruppo di lavoro deve fare parte un'ampia tipologia di membri quali: dirigenti scolastici, docenti, amministrativi, specialisti ed esperti coinvolti nell'insegnamento (psicologi scolastici, consulenti scolastici, etc.), specialisti ed esperti non coinvolti nell'insegnamento (es. psicologi, consulenti, etc.) ed anche i genitori.

La funzione più attiva nel gruppo spetta agli specialisti interni, mentre gli esperti esterni devono aiutare a raggruppare le informazioni necessarie e reperire i materiali necessari, individuare buone pratiche ed, inoltre, formare i docenti e gli specialisti interni. In questo ambito, i genitori sono essenziali, in quanto diventano la "cartina di tornasole" per valutare come vengono accolte e considerate le proposte dai giovani.

Quello che interessa ai ragazzi è l'esigenza di essere riconosciuti nella loro unicità, stante che si trovano in una fase della loro vita dove si fatica a ritrovarsi in una nuova dimensione fisica e mentale che li sta trasformando. Questi giovani chiedono aiuto a fare una sintesi fra le innumerevoli voci che danno consigli, tra i diversi modelli di riferimento, tra le diverse idee. Quello che manca è il dialogo tra tutte le fonti orientative (insegnanti, familiari ed amici, nonché le idee e sensazioni dei ragazzi stessi a proposito dei propri interessi), un supporto che dia la possibilità di far interloquire ed incontrare tutti questi aspetti, soprattutto quando si muovono in direzioni diverse (Bonica, 2011).

"La teoria sociocognitiva attribuisce un ruolo di primo piano al senso di efficacia delle famiglie per quanto riguarda la gestione dei molti aspetti delle relazioni familiari e delle qualità della vita familiare". I genitori motivati da un forte senso di efficacia nella guida dei propri figli, riescono a favorire effettivamente le loro potenzialità; li "difendono con determinazione nelle interazioni con le istituzioni sociali che potrebbero avere effetti importanti su di loro durante il periodo formativo della loro vita" (Bandura, 2012, p. 19).

La famiglia ha un ruolo fondamentale nell'aiutare i ragazzi a conquistare una propria autonomia ma, nello stesso tempo, a garantire loro il migliore futuro realizzabile.

I genitori devono mediare tra il punto di vista del figlio ed i criteri di scelta condizionati dai bisogni della famiglia. 


\section{EDUCARE ALLA SCELTA DELLA SCUOLA SECONDARIA DI SECONDO GRADO E ORIENTARE PER PREPARARE AL FUTURO}

Non bisogna, inoltre, dimenticare che le famiglie ed i ragazzi portano il bisogno di costruire una storia futura coerente con quella passata, ma all'interno di una relazione di fiducia con adulti esperti, come gli insegnanti, possono provare ad ampliare le opzioni e soprattutto l'immaginario rispetto al futuro.

Per ampliare il ventaglio di opzioni è necessaria un'autentica curiosità da parte degli insegnanti per le storie di queste famiglie, una curiosità non giudicante, che colga il sistema di significati che ne guidano le scelte, per poter inserire elementi nuovi.

Gli interventi di orientamento, pertanto, non devono limitarsi a considerare solo i figli, ma devono coinvolgere tutto il nucleo parentale ed incrementare le relazioni positive, non solo fra genitori e figli, ma anche fra famiglie e scuola.

\section{AUTOSTIMA E CONCETTO DI SE'}

E' importante analizzare in che misura le componenti affettivo, emozionali e psicologiche degli allievi, possano influenzare il livello di motivazione, di apprendimento e di autoefficacia nello studio e di successo scolastico.

L'Orientamento scolastico può aiutare in questo, partendo dall'aiuto alla persona, affinché questa acquisisca in maniera autonoma piena conoscenza di sé, così da porre in essere gli opportuni processi decisionali (Del Core, 2008). Se già da adolescenti i ragazzi imparano a conoscere se stessi, accettandosi, con i propri limiti, ed allo stesso tempo, valorizzandoli e tenendo conto delle proprie attitudini e dei propri interessi, diventano autonomi nelle scelte. Questo atteggiamento e approccio ai problemi, servirà per sviluppare un'adeguata capacità progettuale e di decisione, accompagnati dalla conoscenza della realtà sociale e del mondo del lavoro, ma alla base di tutto questo deve esserci la conoscenza di sé (Ibidem).

L'adolescente, pertanto, deve acquisire piena consapevolezza della conoscenza di sé e della realtà esterna.

II docente deve, adoperarsi per: verificare il grado di autostima degli allievi; stimolarli a individuare nella propria esperienza, anche extrascolastica, i successi ottenuti; programmare e calibrare attività che possano far sperimentare un successo ai ragazzi; nella verifica delle attività, cogliere gli aspetti positivi, mentre per gli aspetti negativi, incentrarsi sull'oggetto e non sulla persona (Miceli, 1998).

\section{PROGETTO DI VITA: ORIENTAMENTO E SUCCESSO SCOLASTICO.}

L'orientamento in classe deve indirizzare i ragazzi ad esplorare ed acquisire elementi fondamentali per iniziare la costruzione di una propria identità, partendo dalle competenze acquisite.

Fondamentale diventa l'aiuto a riconoscere le competenze possedute, non solo per sostenere la motivazione ma anche per individuare e definire le scelte formative e di lavoro. È utile nel processo di orientamento, in particolare nella verifica della scelta fatta, riflettere sulle interconnessioni tra caratteristiche personali, specificità del percorso scolastico e caratteristiche delle figure professionali collegate.

La comprensione dei processi decisionali implica alcune domande, come chiedersi quali siano i fattori che determinano una scelta di successo. Naturalmente per conoscere se un fattore è utile oppure no, bisogna verificare se è un elemento che determina la scelta, e se da esso dipende la nostra scelta.

Nel caso di un percorso post-obbligo, per scelta di successo s'intende quella da cui scaturiscono buoni risultati ed un buon grado di soddisfazione, intesa come corrispondenza tra le proprie attese e la realtà. Naturalmente non si possono equiparare le scelte portate comunque avanti, senza interruzioni, con le scelte di successo (scegliere il liceo artistico su consiglio dell'insegnante, perché 
ritiene che sia debole nella materie letterarie, ma in realtà voleva fare lo scientifico, non può essere considerata una scelta di successo, anche se si ottengono buoni risultati, in quanto il livello di soddisfazione sarà basso).

Per un ragazzo assumono particolare importanza i punti di riferimento sui quali può far leva per attingere sicurezza, che dipendono sia da fattori interni (ritenersi valido in una particolare attività 0 capacità interpersonale), sia da fattori esterni (famiglia, insegnanti, compagni). Naturalmente, l'obiettivo non è raggiungere un'alta autostima tout court per ottenere il massimo dei risultati scolastici, ciò sarebbe fuorviante, in quanto se non fondata su risultati reali porterebbe i giovani al rischio di forti delusioni; l'importante è saper autovalutarsi e valutarsi positivamente, per essere consci delle proprie possibilità, in modo da prevedere e aggiustare le aspettative e affrontare le difficoltà in modo costruttivo e fiducioso.

"Uno dei principali obiettivi della scuola è fornire agli studenti gli strumenti intellettivi, le convinzioni e le abilità autoregolatorie che servono loro per autoistruirsi tutta la vita". Imparare ad autogestirsi in situazioni diverse e anche dopo la scuola è il punto di forza che aiuterà gli adolescenti ad essere autonomi e ad "arrivare fino in fondo" (Bandura, 2012, p. 24).

Essenziale diventa per l'adolescente riconoscere se stesso come soggetto qualificato da un capitale di competenze, per poter indirizzare ed utilizzare le proprie energie in esperienze di apprendimento e di attività lavorative, che rendono possibile l'incremento di tale capitale, e sviluppare una rappresentazione della scuola (o meglio, dell'istruzione) legata a significati di espressione e di valorizzazione di sé in una prospettiva di

Gli anni della piena adolescenza sono decisivi per l'esperienza scolastica in quanto, la sua evoluzione inciderà profondamente sul processo di costruzione dell'identità del soggetto. In psicologia dell'adolescenza, i problemi che gli individui devono affrontare in determinati momenti del ciclo di vita, sono determinanti per la crescita. La soluzione appropriata dei problemi favorisce, non solo, l'aumento del senso di empowerment personale, ma consente anche di affrontare con successo i compiti di sviluppo successivi. Per gli adolescenti è molto importante superare positivamente l'insieme dei compiti di sviluppo connessi alla scuola, perché sono già consapevoli del peso che assume per il loro percorso formativo. Tra tutti i compiti di sviluppo dell'adolescente, la scuola viene percepita come una delle esperienze più difficili da affrontare. Tra i compiti di sviluppo del periodo adolescenziale, legati alla scuola, vengono inseriti dagli studiosi il passaggio fra cicli di studio e la scelta del ciclo successivo all'istruzione obbligatoria (Palmonari, 2003).

La transizione fra cicli di studio rappresenta per l'adolescente un momento per verificare le proprie capacità e per superare le difficoltà incontrate, questo compito di sviluppo tranquillizza il soggetto, circa il fatto di essere all'altezza delle richieste poste dalla nuova situazione accrescendo la sua autostima, inoltre, aumenta la fiducia di essere in grado di affrontare con successo gli ulteriori compiti che si presentano.

"Le scelte compiute nei periodi formativi dello sviluppo modellano il corso della vita. Fra le scelte che influiscono sul percorso di vita, quelle che riguardano la carriera e lo sviluppo rivestono un'importanza particolare" (Bandura, 2012, p. 29).

L'adolescente nel decidere il proprio futuro scolastico o lavorativo si trova ad affrontare un processo di riflessione su se stesso, che si va sviluppando durante il corso della vita.

In questa scelta, in altre parole, l'adolescente è obbligato a riflettere su di sé (in termini di caratteristiche personali, di risorse, di competenze, di interessi ed aspirazioni), ed è costretto a chiarire, almeno a se stesso, quali sono le proprie aspettative per il futuro.

II soggetto valuta i propri risultati attraverso l'elaborazione delle proprie esperienze e l'interazione significativa con adulti (gli insegnanti) ed il confronto con alcuni coetanei che mostrano di supe- 
rare con maggiore o minore difficoltà i diversi problemi posti dalla scuola. In tale contesto, l'insuccesso scolastico viene considerato dall'allievo come un rischio che può svalorizzare la propria identità.

L'esperienza scolastica può paragonarsi ad uno specchio strutturato e strutturante, nel quale si può guardare se stessi per valutare ciò che si sa e ciò che si può imparare e specchiarsi nell'immagine del proprio futuro attraverso una presa di coscienza delle proprie risorse e dei propri limiti, in termini di competenze e abilità.

Oggi parlare di orientamento vuol dire proprio "parlare di un nuovo modo di porsi davanti alle cose, affrontando in modo diverso il rapporto tra il proprio essere e l'esterno" (Sgalambro, 2000, p. 123). Affiora quindi in maniera chiara la necessità di considerare nella riflessione sull'orientamento, due aspetti che hanno lo stesso peso: l'identità individuale ed il contesto di riferimento. Su questa relazione si costruiscono le persone e le loro competenze, dal rapporto che si instaura tra sé ed il mondo, dall'equilibrio interno ed esterno che si può interagire coscientemente con la realtà (Ibidem).

L'analisi fin qui svolta, fa emergere alcune conclusioni molto importanti. Innanzitutto, si deve prendere atto che nel mondo contemporaneo si sta affermando la necessità per l'individuo di possedere e di sviluppare un capitale di competenze che gli consentano di impegnarsi ed adattarsi a contesti di lavoro estremamente flessibili. Questo comporta che si sviluppi la capacità di riconoscere le proprie competenze maturate nei diversi ambiti di apprendimento: formale, non formale, ed informale. L'altra conclusione rilevante è che nella società odierna sempre più globalizzata e multiculturale, si devono adottare degli atteggiamenti riflessivi nei confronti delle differenti esperienze, per rispondere all'interrogativo su ciò che si vuole fare della propria vita. Dare una risposta oggi a questa domanda è diventato molto più difficile rispetto alle società tradizionali di una volta, in cui vi erano punti di riferimento fissi. Ma c'è un punto fermo, ovvero il "successo scolastico, che è il riflesso di una costellazione di competenze personali, ed è un buon predittore del successo lavorativo" (Bandura, 2012, p. 33).

Ecco che tutto si muove attorno all'Orientamento che fa da volano per il successo scolastico e formativo, per realizzare un progetto più ambizioso, che è il progetto di vita.

\section{BIBLIOGRAFIA}

Bellamì, D. \& Scandella, O. in AA.VV. (2002). La scuola che orienta. Milano: La nuova Italia.

Bonica, L. (2011). Circoli virtuosi e circoli viziosi, lin L. Bonica, M. Olagnero (a cura di) Come va la scuola? Genitori e figli di fronte a scelte e carriere scolastiche. Roma: Infantiae.Org.

Bracken, B.A. (1992). MSCS-Multidimensional Self-Concept Scale. Austin (Texas): RP_ED. Trad. it. Testi di valutazione multidimensionale dell'autostima. Trento: Edizioni Erickson, 2003.

Cornaviera, D. (2008). La relazione tra didattica e orientamento. lin Focciatti, R. (a cura di). Orientare e orientarsi nella scuola primaria e secondaria. Roma: Carocci.

Del Core, P. (2008). Orientamento: strumento chiave per educare alle scelte. Roma: Anicia.

Mancinelli, M.R. e Manto, M.C. (2004). I bisogni orientativi in età evolutiva., lin Castelli, C. (a cura di). Orientamento in età evolutiva. Milano: Franco Angeli.

Marostica, F. (2003). Orientamento e sistema scolastico: una via emiliana? Una scuola allo specchio. Rapporto regionale 2003., USR e IRRE Emilia Romagna. Bologna: Editcomp.

Marostica, F. (2008) (a cura di Pombeni, M.L.). La centralità della didattica orientativa nel processo di sostegno all'autoorientamento. L'orientamento tra passato e futuro: l'esperienza di Bologna. Roma: Carocci

Miceli, M. (1998). L'autostima. Bologna: II Mulino.

MIUR 19 febbraio 2014, Linee guida nazionali per l'orientamento permanente. 
Morin, E. (2000). Una testa ben fatta. Milano: Raffaello Cortina Editore.

Nicoli, D. (2008). II modello comune di didattica delle competenze in Alternanza Scuola Lavoro e didattica per competenze: per una formazione efficace. Esperienze del Veneto 2007-2008, Regione del Veneto. Mirano: Grafiche Serenissima.

Palmonari, A. (1993). Psicologia dell'adolescenza. Bologna: Il Mulino.

Pellerey, M.(2004). Le competenze individuali e il Portfolio. La Nuova Italia: Milano.

Pombeni, M. L. (1996). Orientamento scolastico e professionale. Bologna: II Mulino.

Pope, A., Craighead, E., e McHale S (2008). Migliorare l'Autostima. Un approccio psciopedagogico per bambini ed adolescenti. Trento: Erickson.

Scandella, 0. (2002). in La scuola che orienta: percorsi, ruoli, strumenti. Firenze: La nuova Italia.

Sgalambro, L. (2000). Scuola, orientamento e lavoro, Roma: Carocci editore.

Soresi, S. (2007). Orientamento alle scelte: rassegne, ricerche, strumenti ed applicazioni. Firenze: Giunti.

Van Esbroeck. R. (2011). Career guidance in secondary education: a model. Rivista "Counseling", 4(2), Trento: Erickson, 127-128.

Varisco, B.M. (2004). Portfolio. Valutare gli apprendimenti e le competenze. Roma: Carocci.

Viglietti, M. (1990). La metodologia dell'orientamento. Orientare chi, come, perché. Tornino: SEl.

Zanniello, G. (2003). Didattica orientativa. Una metodologia educativa per l'attivazione dello sviluppo professionale e personale. Napoli: Tecnodid. 
